\title{
FONDEMENTS D'UNE SOCIOLOGIE ECONOMIQUE DE L'ENVIRONNEMENT
}

\author{
Corinne Gendron
}

Presses de Sciences Po | « Ecologie \& politique »

2003/1 N²7 | pages 59 à 78

ISSN 1166-3030

ISBN 9782847970665

Article disponible en ligne à l'adresse :

https://www.cairn.inforevue-ecologie-et-politique1-2003-1-page-59.htm

Distribution électronique Cairn.info pour Presses de Sciences Po.

(C) Presses de Sciences Po. Tous droits réservés pour tous pays.

La reproduction ou représentation de cet article, notamment par photocopie, n'est autorisée que dans les limites des conditions générales d'utilisation du site ou, le cas échéant, des conditions générales de la licence souscrite par votre établissement. Toute autre reproduction ou représentation, en tout ou partie, sous quelque forme et de quelque manière que ce soit, est interdite sauf accord préalable et écrit de l'éditeur, en dehors des cas prévus par la législation en vigueur en France. Il est précisé que son stockage dans une base de données est également interdit. 


\section{FONDEMENTS D'UNE \\ SOCIOLOGIE ECONOMIQUE \\ DE L'ENVIRONNEMENT}

Corinne Gendron •

厂

9 industrie occupe un rôle particulier dans la crise mondialisée de l'environnement ${ }^{1}$. Première au banc des accusés, elle demeure, dans l'esprit de la population, la principale responsable de la dégradation des écosystèmes. Pourtant, après une longue période de dénégation, le discours des entreprises a radicalement changé au cours des années 1980 et les initiatives industrielles en faveur de l'environnement se sont multipliées. Un nombre croissant d'entreprises adhèrent à des codes de conduite environnementaux, et on compte pas moins de 40000 entreprises certifiées ISO 14001 à travers le monde. Les dirigeants d'entreprises ont aussi pris une part active aux délibérations de la conférence de Rio en 1992. À titre de signataires du rapport Changer de Cap du Business Council for Sustainable Development, ces dirigeants se sont personnellement engagés à promouvoir le développement durable dans le cadre d'un partenariat inédit avec les autres acteurs de la sociétée

Corinne Gendron est professeur à l'Université du Québec à Montréal (Canada).1 Nous tenons à remercier les organismes suivants sans le soutien desquels nous n'aurions pu mener à terme nos recherches : CRSH, FCAR, Fondation Desjardins, FCAR-MEQ-MRI. La thèse de doctorat dont cet article est largement inspiré a été couronnée par le Prix de l'Institut de la recherche en économie contemporaine (IRÉC) 2001.

2 Stephan Schmidheiny, Changer de cap : réconcilier le développement de l'entreprise et la protection de l'environnement, Dunod, Paris, 1992. 
Mais plusieurs estiment que la portée réelle de ces initiatives managériales en regard de la préservation de l'environnement demeure limitée car le système économique empêche les entreprises de véritablement prendre en considération les contraintes écologiques. Issu d'une époque où les systèmes de production n'avaient que peu de répercussions sur le milieu, le système économique actuel favorise la surexploitation des ressources et la production de pollution en excluant de son cadre conceptuel la dynamique et les contraintes écologiques. L'économie s'est constituée comme sphère autonome non seulement par rapport au social et au politique mais aussi par rapport à l'environnement qu'elle a marchandisé et démembré en « ressources naturelles » ${ }^{3}$. C'est à cet impair historique ayant présidé à une véritable schizophrénie écologique de l'économie que les économistes de l'environnement tentent de remédier par un ré-arrimage des disciplines de l'économie et des sciences naturelles.

Dans cet article, nous explorons les propositions des économistes de l'environnement à la lumière d'une approche de sociologie économique et d'une recherche sur le terrain que nous avons menée pendant les années 1999 et 2000 auprès des hauts dirigeants du Québec. Nous présentons tout d'abord les différents courants économiques s’intéressant à l'environnement, pour proposer ensuite un éclairage nouveau sur la dynamique de la modernisation écologique des institutions économiques. Dans un troisième temps, nous dévoilons les résultats de notre recherche en montrant comment ils répondent aux différentes propositions des économistes de l'environnement.

\section{L'économie et l'environnement}

Participant d'un véritable processus d'autonomisation par rapport aux cycles et aux contraintes naturels, le formalisme économique illustre l'affranchissement que semblait désormais garantir le pouvoir formidable d'une industrialisation émancipatrice. Ainsi, au cœur même du formalisme néoclassique est inscrit l'idéal du progrès de la société industrielle : la production de biens, en abondance suffisante pour éliminer la pertinence des questions de distribution et restreindre la

3 John Barry, Environment and social theory, Routledge, Londres et New York, 1999. 
nécessité du recours à un ordre politique potentiellement arbitraire, où une nature instrumentalisée et maîtrisée est mise au service de cette surproduction. La dissociation écologique de l'économie a suscité des questionnements précoces chez les économistes, qu'il s'agisse de Smith, Mill, Riccardo ou Malthus ${ }^{4}$. Mais ce n'est qu'aujourd'hui, alors que les dysfonctionnements écologiques ont acquis une dimension systémique et ne se posent plus simplement en termes de rareté, mais de rupture des grandes régulations écologiques, que cette discipline se penche enfin sur les conséquences d'un système économique désencastré de la biosphère. Pour les économistes préoccupés par la dégradation de l'environnement, c'est bel et bien l'aveuglement et la méconnaissance qu'a l'économie des cycles et des limites écologiques qui sont à l'origine de la crise actuelle. En s'autonomisant des écosystèmes, l'économie a démembré la nature en ressources naturelles et a développé une vision essentiellement instrumentale de la nature. Appréhendées comme intrants dans un système de production canalisant la majeure partie de l'énergie sociale, on ne s'est guère penché sur la capacité de régénération et la disponibilité des ressources de l'environnement. Le caractère clos du système de production interdisait aussi la reconnaissance des interactions de la production avec le milieu récepteur en fin de cycle, c'est-à-dire les limites de la capacité d'assimilation des déchets et des émissions par les écosystèmes. Pour les économistes de l'environnement, la crise écologique est donc la conséquence directe d'un formalisme économique tronqué des réalités écologiques, d'une économie pensée comme système clos et indépendant du substrat naturel de la vie et des activités humaines. Il est donc impératif de corriger les aberrations d'une évolution historique aux conséquences néfastes en reconstruisant le lien brisé, mais nécessaire, entre économie et environnement.

Ce défi a suscité des réponses différentes au sein d'écoles de pensée plus ou moins orthodoxes dont les propositions sont intimement liées au diagnostic qui est fait de la problématique environnementale. À l'extrême de l'orthodoxie, l'école des droits de propriété postule que la crise écologique résulte d'une structure inadéquate des droits de propriété, et qu'il suffit donc de privatiser l'environnement pour assurer l'utilisation

4 Jean-Philippe Barde et Gerelli Emilio, Économie et politique de l'environnement, PUF, Paris, 1977 ; Robert Costanza, John Cumberland, Herman Daly, Robert Goodland et Richard Norgaard, An introduction to ecological economics, St. Lucie Press, Florida, 1997. 
efficiente des ressources ${ }^{5}$. D'inspiration coasienne, ce courant adhère à l'idéologie d'un État minimal et avance que la protection de l'environnement ne requiert qu'un seul acte gouvernemental : la privatisation. L'argumentation de l'école néoclassique repose plutôt sur l'idée que l'environnement est sur-utilisé parce qu'il est sous-évalué. Cette sous-évaluation est due aux caractéristiques particulières des biens communs dont l'environnement est un exemple typique, et requiert une intervention gouvernementale permettant de révéler le véritable coût des biens, tel que le suggérait Pigou en 1920. Il ne s'agit pas tant de révéler un marché préexistant comme l'envisage l'école des droits de propriété, que de simuler une dynamique de marché permettant de gérer les ressources naturelles d'une manière efficiente. De façon générale, les propositions de l'école néoclassique appellent une internalisation des externalités.

On peut distinguer deux branches dans l'école néoclassique que nous proposons de désigner comme suit : les versions radicale et appliquée. La version radicale de l'école néoclassique s'emploie à déterminer un taux de dépollution (ou de pollution) optimal grâce à l'outillage traditionnel de la micro-économie. En fondant le niveau de dépollution souhaitée sur le consentement à payer, la méthodologie retenue permet d'obtenir un optimum parétien se situant à l'intersection des courbes d'offre (équipement anti-pollution) et de demande (valeur agrégée des biens environnementaux estimée à partir du consentement à payer). $\mathrm{Ce}$ marché fictif permet d'établir un prix des biens environnementaux qui, en se substituant à la gratuité actuelle, permettrait de stopper leur surutilisation. Cette démarche soulève deux objections majeures. D'une part, il est admis depuis longtemps que la valeur des biens communs, dont l'environnement est un exemple type, ne peut être obtenue à partir des méthodologies reposant sur le consentement à payer ${ }^{6}$. D'autre part, le niveau optimal de pollution ou de dépollution calculé à partir de valeurs économiques n'a aucune base écologique, et s'avère totalement déconnecté des réalités biophysiques. Outre le fait que l'optimum économique est indépendant des phénomènes de seuil écologique, Pearce

5 Michael Jacobs, The green economy. Environment, sustainable development and the politics of the future, UBC Press, 1993 ; Michael Jacobs, « The limits to neoclassism », in Michael Redclif et Ted Benton (dir.), Social theory and the global environment, Routledge, Londres et New York, 1994, p. 67-91.

6.Michael Jacobs, 1993 et 1994, op. cit. 
démontre qu'un optimum économique obtenu à une période donnée entraîne nécessairement un sous-optimum écologique à la période suivante, c'est-à-dire une dégradation de l'environnement, tandis qu'un optimum écologique fixé a priori s'accompagne d'un sous-optimum économique 7 . Bref, la prétention de l'école néoclassique radicale se heurte aux limites des outils économiques incapables de traduire les contraintes et la dynamique écologiques réelles.

La branche appliquée de l'école néoclassique admet ces limites quant à la détermination d'un niveau de pollution (ou de dépollution) approprié, et reconnaît la pertinence d'un processus décisionnel politique et scientifique en la matière. Sa proposition vise plutôt l'utilisation d'instruments économiques dans la mise en cuvre des politiques publiques de protection de l'environnement. Plus flexibles, moins coûteux, incitatifs plutôt que contraignants et décentralisés, ces instruments tirent profit de la dynamique du marché et mettent son potentiel régulateur au service de la protection de l'environnement. L'instauration des principes d'utilisateur-payeur et de pollueur-payeur, la mise en œuvre de marché de droits de pollution de même que les taxes écologiques sont présentées comme des alternatives efficientes à une réglementation tatillonne et dépassée. Pourtant, l'expérience démontre que plusieurs avantages présumés des instruments économiques ne se vérifient guère dans la pratique.

Les cas où l'on a pu observer une dynamique incitative sont rares, et les coûts d'application sont tout aussi élevés, sinon plus que ceux de la réglementation traditionnelle. Si bien que l'efficacité supérieure des instruments économiques reste à démontrer. Mais de manière plus fondamentale, la contradiction qu'opère l'école néoclassique appliquée entre la réglementation et les outils économiques de protection de l'environnement repose sur une lourde confusion quant à leur nature respective qui n'est pas étrangère au débat sur la déréglementation qui anime nos sociétés depuis les vingt dernières années. Alors qu'ils sont présentés comme une alternative réglementaire, les outils économiques correspondent plutôt à une nouvelle forme de réglementation ayant potentiellement pour effet de faire basculer la protection de l'environnement du domaine judiciaire au domaine administratif, et par 
conséquent de décriminaliser ce champ de régulation sociale. Enfin, la perspective de l'école néoclassique appliquée appréhende la réglementation comme un facteur exogène, et son analyse ne fait aucune place à la dynamique réelle des acteurs sociaux dans le processus d'élaboration des normes environnementales auxquels ils sont assujettis. Or, ce processus d'élaboration se présente comme une dynamique complexe entre le public, les autorités réglementaires et les acteurs réglementés où les rapports de compétitivité et le développement technologique qui leur est intrinsèquement lié s'avèrent sans doute plus déterminants qu'une quelconque efficacité économique, ou même écologique.

L'approche néoclassique comporte un troisième volet sans véritable lien avec les deux précédents qui nous transporte dans la dimension macroéconomique de la problématique environnementale. L'approche néoclassique macro-économique propose une conception de la production et du développement qui reconnaît l'existence du facteur écologique, sans toutefois l'ériger en contrainte pour le développement. Cherchant à intégrer les ressources naturelles à la fonction de production, la variante Solow-Stiglitz suppose que tout output constitue nécessairement une combinaison de capital, de travail et de ressources, mais n'interdit pas que l'apport de ces dernières soit infiniment petit. Envisagé dans la perspective néoclassique, le développement durable requiert simplement le maintien d'un stock de capital total constant, ce qui autorise une substitution des différentes formes de capital (naturel, financier et manufacturé) et repose sur l'idée d'une trajectoire optimale d'épuisement des ressources (scénario dit de soutenabilité faible). Ces théories conservatrices sont vertement critiquées par l'école américaine qui s'inscrit dans le courant plus large de l'économie écologique.

En marge de l'économie de l'environnement qu'elle accuse d'économiser l'environnement plutôt qu'elle n'écologise l'économie, l'économie écologique se présente comme une nouvelle discipline issue à la fois de l'économie et de l'écologie ${ }^{8}$. Pour ce courant, la problématique environnementale résulte principalement d'un conflit entre deux logiques, l'une linéaire, illimitée et déterministe, l'autre circulaire,

8 Herman E. Daly, « Free market environmentalism : turning a good servant into a bad master », Critical Review, vol. 6, $\mathrm{n}^{\circ} 2-3,1993$, p. 171-83 ; Herman E. Daly, Ecological economics and the ecology of economics. Essays in criticism, Edward Elgar, Cheltenham, 1999. 
limitée et chaotique, qu'il importe d'arrimer en vue de maîtriser les conséquences écologiques des activités économiques. Dans sa volonté de reconnaître la dépendance de l'économie vis-à-vis de l'environnement, l'école de Londres insiste sur la multifonctionnalité de ce dernier qui ne peut être réduit à des ressources ou à un puits assimilateur, mais fournit également des biens naturels, ainsi que la fonction plus générale de support à la vie (life support). Cette école rejette également l'hypothèse de substituabilité illimitée des différentes formes de capital. Toutefois, bien qu'en rupture avec la pensée néoclassique traditionnelle sur ces points fondamentaux, la méthodologie économiste et monétaire privilégiée par l'école de Londres réduit la portée novatrice de ses propositions et la confine au formalisme néoclassique dont elle prétend s'émanciper. Ainsi, bien qu'elle se présente comme un courant radical, l'école de Londres partage de nombreux points communs avec l'analyse et les propositions néoclassiques.

À l'inverse, l'école américaine, tout en cherchant l'adhésion caractéristique d'un courant largement répandu, propose une révision $d u$ formalisme macro-économique qui implique une rupture fondamentale avec les principes au cœur même du système capitaliste. L'appel à une croissance zéro repose sur l'idée qu'un sous-système ne peut croître indéfiniment si le système dans lequel il est inclus est clos. En d'autres termes, l'hypothèse d'une croissance économique illimitée n'est pas viable compte tenu du caractère fini de la planète. Le problème écologique se pose donc essentiellement en termes de taille de l'économie. Le programme de l'école américaine ouvre donc le débat sur la croissance économique qui est certainement l'un des plus controversés actuellement dans le domaine de l'environnement. À l'instar de Berger, nous soutenons néanmoins que ce n'est pas tant la taille de l'économie que son intensité écologique qui pose problème pour la biosphère ${ }^{9}$. Ceci ne signifie pas que la croissance économique n'entraîne pas une dégradation accrue de l'environnement, mais plutôt que cette dégradation résulte de l'ancrage écologique particulier de l'économie industrielle dont la croissance vient amplifier les effets. L'hypothèse d'une croissance qui n'aggrave pas la dégradation en cours est donc théoriquement possible, mais suppose une baisse de l'intensité 
écologique, ou dématérialisation de l'économie par laquelle le niveau d'activité se trouve dissocié du degré de ponction exercé sur l'environnement. Par ailleurs, le scénario de la dématérialisation s'avère tout aussi nécessaire dans le cadre d'une économie stationnaire dont on chercherait à réduire l'impact écologique en deçà des limites de la capacité de charge de la planète ${ }^{10}$.

L'hypothèse de la dématérialisation de l'économie repose sur le potentiel d'un développement technologique susceptible de réduire substantiellement l'intensité écologique de l'économie (tant dans sa dimension productive que consommatoire). En tenant compte des tendances démographiques et du taux de croissance de la consommation, Ehrlich et Ehrlich expliquent qu'une réduction de l'impact écologique de cinquante pour cent suppose une dématérialisation de quatre-vingt-onze pour cent, ce qui semble nettement irréaliste ${ }^{11}$. Mais au-delà du degré d'effort requis, on peut également s'interroger sur le potentiel réel de la technologie dès lors qu'on ne la conçoit plus comme simple instrumentalité d'une volonté publique. En effet, le développement technologique est modelé par des rapports sociaux dont la dynamique oriente les finalités et marque l'évolution. Si bien que la technologie ne peut être envisagée comme une solution exogène susceptible d'enrayer un phénomène auquel elle participe. Le développement technologique n'est pas plus neutre sur le plan social qu'il ne l'est sur le plan écologique et l'appréhender selon un schéma causal déterministe où elle tient le rôle de moyen reflète une profonde incompréhension de sa dynamique.

Ces dimensions oubliées de la technologie tout comme la formalisation technique du rapport économie-environnement ou encore la conception réductrice du processus de régulation politique font ressortir les limites des approches économiques de l'environnement qui sont incapables de rendre compte des dynamiques sociales, que celles-ci se manifestent au niveau de la crise écologique ou du système économique ${ }^{12}$. En d'autres termes, les principales approches économiques d'environnement ne 10 Ibid.

11 Paul R. Ehrlich et Anne H. Ehrlich, The population explosion, Simon and Schuster, New York, 1990.

12 Olivier Godard et Jean-Michel Salles, «Entre nature et société. Les jeux de l'irréversibilité dans la construction économique et sociale du champ de l'environnement », in Robert Boyer, Bernard Chavance et Olivier Godard (dir.), Les figures de l'irréversibilité en économie, Éditions de l'EHESS, Paris, 1991, p. 233-272 ; Olivier Godard, «L'écodéveloppement revisité », Économies et Sociétés, série « Développement, croissance et progrès », F, n 36, 1/1998, p. 213-229. 
reconnaissent pas plus le caractère socialement construit de l'économie que le caractère socialement construit de la problématique environnementale. La nouvelle approche institutionnaliste de l'environnement propose bien quelques pistes en insistant sur le rôle de médiation entre l'humain et la nature que jouent les institutions sociales, et en plaidant pour une réforme institutionnelle qui intègre et reflète les conditions et les contraintes qu'imposent la biosphère et ses grandes régulations aux activités humaines. Néanmoins, ce courant encore embryonnaire n'offre pas véritablement de cadre analytique pour penser la modernisation écologique et la dynamique sociale qui la sous-tend tandis que ses propositions appartiennent encore au registre des exhortations. Dans d'autres travaux récents, des chercheurs régulationnistes soulignent aussi les carences des approches économiques de l'environnement incapables de reconnaître le caractère socialement construit non seulement de la problématique environnementale, mais également du système économique ${ }^{13}$. Or, dans une perspective socio-économique résolument hétérodoxe, le cadre régulationniste classique permet au contraire de penser la dimension construite des institutions économiques et propose une théorie féconde des crises et des transformations qu'a connues le capitalisme depuis ses débuts.

\section{Théoriser la dimension sociale des transformations économiques}

Un modèle de développement capitaliste peut entrer en crise pour diverses raisons, mais l'originalité de l'approche de la régulation est d'envisager l'existence de crises endogènes, c'est-à-dire inhérentes au fonctionnement même du système. Ainsi, pour les régulationnistes, crises et périodes de stabilité ne sont que les deux faces de la régulation sociale : l'une exprime alors que l'autre contient la conflictualité originaire des rapports sociaux ${ }^{14}$. La crise économique qui s'est ouverte

13 Joachim Becker et Werner G. Raza, « Theory of regulation and political ecology : an inevitable separation ? », Économies et Sociétés, série « Théorie de la régulation », R, n 11, 1/ 2000, p. 55-70.

14 Alain Lipietz, « Accumulation, crises et sorties de crise : quelques réflexions méthodologiques autour de la notion de "régulation" ", Long waves, crises and technological, social and political development, $\mathrm{n}^{\circ} 8409$, Helsingor, 2-4 mars 1984, p. 8. 
avec les années 1970 correspond précisément à la rupture du grand compromis fordiste entre les gestionnaires et les travailleurs supporté par l'État providence. Elle s'est accompagnée d'un effritement irrémédiable du projet industriel mis en cause par des mouvements sociaux qui contestent l'idée d'un progrès social pensé en termes de science et de technologie, de consommation et de redistribution administrative de l'accumulation. Comme issue à cette crise, l'approche régulationniste anticipe la formation d'un nouveau grand compromis social donnant lieu à des institutions sur la base desquelles pourrait s'échafauder un modèle de développement capitaliste inédit porteur d'une période de prospérité comparable aux trente glorieuses.

La conclusion d'un tel compromis est inévitable dans la mesure où de façon générale, un acteur dominant est incapable d'imposer totalement ses vues aux autres acteurs sociaux ; il est donc forcé de faire certaines concessions en vue d'atteindre ses objectifs ${ }^{15}$. À défaut d'un compromis, la lutte se poursuit et aucune des parties n'est satisfaite alors qu'elles se consument dans le conflit. Le compromis institutionnalisé permet de fixer certaines règles qui orientent et guident l'action économique et sociale pour une période donnée, jusqu'à ce que s'aggravent les tensions non résolues par le compromis au point de le déstabiliser ${ }^{16}$. L'école régulationniste s'est donc concentrée sur la configuration possible d'un grand compromis post-fordiste. Cependant, sa conception restrictive du social confine ses recherches au domaine du travail et de la production, et l'empêche de tirer pleinement profit de son cadre théorique pour penser les transformations en cours ${ }^{17}$. En effet, contrairement à ce que suggère la pensée marxiste dont s'inspire la théorie de la régulation, les rapports sociaux sous-jacents aux crises dépassent le champ du travail et ne se réduisent pas aux seuls rapports de production. En se focalisant sur la dimension du travail, les régulationnistes répondent certes à l'agenda politique de la plupart des pays industrialisés dans le contexte de la crise économique, mais font l'impasse sur plusieurs transformations essentielles du modèle de développement, dont la question écologique qui ne cesse pourtant de prendre de l'importance depuis les trente

15 Robert Delorme et André Christine L'État et l'économie. Un essai d'explication de l'évolution des dépenses publiques en France, 1970-1980, Éditions du Seuil, Paris, 1983.

16 Alain Lipietz, op. cit. ; Alain Touraine, Production de la société, Éditions du Seuil, Paris, 1973, p. 218.

17 Paul R. Bélanger et Benoît Lévesque, « La "théorie" de la régulation, du rapport salarial au rapport de consommation. Un point de vue sociologique », Cahiers de recherche sociologique, $\mathrm{n}^{\circ}$ 17, 1991, p. 15-51. 
dernières années. À cet égard, la théorie de la régulation s'avère aussi peu novatrice que les grandes approches néoclassiques traditionnelles qui sont la cible des économistes de l'environnement. En vue de remédier à ces carences, des recherches récentes proposent d'intégrer au cadre régulationniste une sixième forme institutionnelle permettant de caractériser un régime d'accumulation en fonction de son degré d'intensité écologique ${ }^{18}$. À l'instar des autres formes institutionnelles, la contrainte écologique est au cœur de conflits sociaux susceptibles de donner lieu à des compromis institutionnalisés. Mais pour penser ces compromis, il est indispensable d'élargir la conception régulationniste du social de manière à inclure la dynamique sociale entourant la question de l'environnement.

Tout d'abord, il importe de rappeler que contrairement à ce que laisse entendre Lipietz, un modèle de développement post-fordiste écologiste ne suppose pas la conclusion d'un compromis institutionnalisé avec la nature ${ }^{19}$. Une relation sociale implique nécessairement des acteurs $\operatorname{sociaux}^{20}$, si bien que la relation humain/nature ne peut être appréhendée comme un rapport social en soi. La problématique environnementale doit plutôt être envisagée comme un champ à l'intérieur duquel des acteurs sociaux en conflit participent inégalement à une intervention sur l'organisation sociale. Si bien qu'un éventuel modèle de développement post-fordiste écologiste ne reposera pas sur un compromis avec la nature, mais plutôt sur un compromis au sujet de la nature, sur la base duquel sera établi un schéma particulier d'interaction entre les humains et les écosystèmes conforté par une représentation de la nature largement partagée. C'est au niveau de cette représentation qu'intervient la transformation de la relation humain/nature qui vient bousculer la vision fordiste du progrès. Si l'on étend au domaine de l'environnement l'articulation entre l'économique et le social que propose l'école de la régulation dans le domaine du travail, il est nécessaire de compléter notre portrait économique par une compréhension de la dynamique sociale

18 Christoph Görg, «The regulation of the societal relationships with nature - some theoretical remarks », communication à Transitions towards a sustainable Europe. Ecology-economy-policy, 3rd Biennal Conference of the European Society for Ecological Economics, Vienne, 3-6 mai 2000 ; Joachim Becker et Werner G. Raza, op. cit.

19 Alain Lipietz, "Écologie politique régulationniste ou économie de l'environnement ? ", in Robert Boyer et Yves Saillard (dir.), Théorie de la régulation. L'état des savoirs, La Découverte, Paris, 1995, p. 351.

20 Alain Touraine, «Théorie et pratique d'une sociologie de l'action », Sociologie et sociétés, vol. X, n 2, 1978, p. $149-188$. 
propre au champ de l'environnement. Alors que les théories économiques envisagent la crise écologique comme une série de faits et de données, les approches sociologiques insistent sur le caractère construit de la problématique environnementale. Cette construction à la fois sociale et scientifique repose sur une dynamique complexe et conflictuelle entre les acteurs sociaux, si bien que la montée des préoccupations écologiques ne peut être appréhendée comme une conséquence directe et linéaire de la dégradation matérielle de l'environnement ${ }^{21}$. Il importe donc de comprendre à travers quel processus la question environnementale a pu s'imposer comme enjeu à l'échelle de la société.

Pour ce faire, nous nous sommes penchés sur les différentes théorisations du phénomène de mobilisation collective qui ont été élaborées depuis le milieu du siècle dernier. La première école dite du comportement collectif proposait une analyse psychosociologique des mouvements sociaux. Ceux-ci étaient envisagés comme des réponses irrationnelles à la modernisation sociale. Non institutionnelle par définition, l'action collective était le résultat d'une dynamique d'agrégation des frustrations individuelles évoluant de la foule à des formes d'action plus organisées. Le deuxième temps de la pensée de l'action collective est marqué par une théorie hyperrationnelle aux antipodes de cette première école. La logique de l'action collective envisagée par Olson propose une lecture économiste qui brise le mythe du caractère naturel de la mobilisation sociale. Bien qu'elle ignore les déterminants sociaux de l'action collective qu'elle appréhende uniquement en termes rationnels, elle a eu le mérite de faire ressortir le fait que la mobilisation n'est pas un comportement automatique ou un phénomène qui va de soi, bref qu'elle doit être expliquée ${ }^{22}$. L'école de la mobilisation des ressources ouvre, avec l'école des nouveaux mouvements sociaux, le troisième et dernier temps de l'analyse des mobilisations collectives. Largement inspiré par la perspective olsonnienne, l'école de la mobilisation des ressources a hérité de sa perspective instrumentale même si elle s'est efforcée de dépasser le défi posé par le paradoxe d'Olson ${ }^{23}$. En déplaçant l'objet de recherche du pourquoi vers le comment, cette école apportera un éclairage indispensable sur le fonctionnement et l'organisation de la mobilisation

21 Johannes Berger, op. cit.

22 Érik Neveu, Sociologie des mouvements sociaux, La Découverte, Paris, 1996.

23 Mancur Olson, Logique de l'action collective, PUF, Paris, 1978. 
collective. Elle dépassera également l'opposition factice entre action institutionnelle et action non-institutionnelle en développant une conception instrumentale où la mobilisation collective est orientée vers l'institutionnalisation, c'est-à-dire vers la reconnaissance et la consécration politique d'acteurs et d'intérêts nouveaux. Le modèle de McCarthy et Zald s'inspire de la configuration du marché pour brosser un portrait de la structure et de la dynamique des organisations en environnement ${ }^{24}$. Leurs analyses font ressortir le caractère stratégique et organisé d'une action collective qui ne peut plus être pensée comme irrationnelle et spontanée. Oberschall tente pour sa part d'ancrer davantage les phénomènes de mobilisation dans le tissu social qui peut être plus ou moins porteur de mobilisation ${ }^{25}$. Son modèle établit une typologie où le potentiel de mobilisation s'explique en fonction de l'accès aux structures de pouvoir, d'une part, et du type de solidarité existant dans le groupe sujet à mobilisation, d'autre part. Enfin, la perspective historique de Tilly permet une avancée notable dans la compréhension du phénomène de mobilisation : les concepts de registres et de répertoires redonnent à l'analyse une dimension sociologique indispensable qui permet d'appréhender l'action collective comme un fait proprement social répondant à une dynamique particulière ${ }^{26}$.

De façon générale toutefois, l'école de la mobilisation des ressources ne réussira jamais à reconnaître entièrement la nature sociale du phénomène de l'action collective et ses implications au plan analytique. Enfermée dans sa perspective utilitariste, elle se prive ainsi d'une gamme de signifiants irréductibles à la logique économiste d'une fonction maximisatrice. Enfin, elle ne réussit pas à rendre compte des mobilisations qui émergent à partir de la fin des années 1960 ; celles-ci ne répondent guère à un schéma d'action utilitariste orienté vers l'institutionnalisation et appellent une perspective inédite libérée des postulats comportementaux économistes. L'école des nouveaux mouvements sociaux fera justement de ces mobilisations atypiques son principal objet d'étude. Dans une perspective résolument sociologique s'ouvrant sur l'analyse plus large des transformations de la société industrielle, les nouveaux mouvements sont appréhendés comme la 24 John D. McCarthy et Mayer Zald, «Ressource mobilization and social movements : a partial theory », American Journal of Sociology, vol. 82, $\mathrm{n}^{\circ}$ 6, 1977, p. 1212-1241.

25 Anthony Oberschall, Social conflict and social movements, Prentice Hall, Englewood Cliffs, 1973.

26 Charles Tilly, From mobilization to revolution, Addison-Wesley Pub., Reading, 1976. 
manifestation d'un nouveau type de société, ou à tout le moins d'une nouvelle configuration de l'ordre socio-politique. L'action collective typique des dernières décennies devient l'occasion d'élaborer une nouvelle conception de la société et même une nouvelle sociologie ${ }^{27}$. Pour Touraine, cette nouvelle sociologie doit délaisser les structures pour enfin se tourner vers l'action sociale car la société n'est pas que reproduction, elle est aussi production conflictuelle d'elle-même. Ce sont les acteurs sociaux qui, à travers leurs luttes pour le contrôle de l'historicité, interviennent sur l'organisation sociale. L'historicité, c'està-dire l'ensemble des formes de travail que la société exerce sur ellemême, se traduit par un mode d'investissement, un mode de connaissance ainsi qu'un mode culturel. Le mode d'investissement concerne l'affectation de la partie non consommée du produit pour intervenir sur l'organisation économique. Le mode culturel touche la conception de la créativité sociale d'une société. Enfin le mode de connaissance traduit le rapport d'une société à son environnement. La société post-industrielle en émergence se démarque fondamentalement de la société industrielle qui la précède par son mode d'investissement tout d'abord, qui vise la transformation de la finalité de la gestion de la production plutôt que seulement la consommation, la distribution et l'organisation. Mais elle se distingue aussi par un mode culturel réflexif, c'est-à-dire une conception réflexive de la créativité sociale où la société se perçoit comme se produisant elle-même, de même qu'un mode de connaissance moins déterministe qui fonde l'idée d'une société responsable d'elle-même. L'émergence de nouveaux mouvements sociaux marque l'avènement de cette société industrielle aux caractéristiques distinctives. Ils témoignent du déplacement de l'antagonisme central du système historique de la société industrielle (capitalistes-travailleurs) vers un antagonisme propre au système historique de la société post-industrielle (technocrates-usagers). Ainsi, pour Touraine, la diversité des nouveaux mouvements sociaux n'est qu'une étape transitoire devant mener à la stabilisation d'un nouveau couple antagonique caractéristique de la société post-industrielle, et ses travaux visent à identifier le mouvement social qui jouera le rôle que jouait le mouvement ouvrier dans la société industrielle. S'il adhère à 
l'idée d'une société post-industrielle, Melucci préfère toutefois reconnaître dans la multiplicité des mouvements non pas une simple étape transitoire mais la caractéristique même de ce nouveau type de société $^{28}$. L'action sociale y est portée par un réseau de mouvements plutôt que par un couple de classes antagoniques. Offe envisage pour sa part une configuration socio-politique où les nouveaux mouvements sociaux viennent se juxtaposer à l'ancienne structure bipolaire de la société industrielle, autorisant une série d'alliances plus ou moins porteuses de transformations ${ }^{29}$. Contrairement à l'école de la mobilisation des ressources toutefois, ces transformations ne sont pas simplement envisagées comme une institutionnalisation des acteurs et de leurs intérêts à l'intérieur d'un système politique statique. Les nouveaux mouvements constituent plutôt un défi à l'ordre institutionnel actuel en politisant des thèmes à la frontière des sphères publique et privée, formulés en termes d'enjeux non négociables, par des acteurs mal définis engagés dans des luttes à caractère universel. Il est d'ailleurs trompeur de parler d'institutionnalisation car l'arrivée de ces nouveaux mouvements sociaux vient transformer l'espace public; en d'autres termes, plutôt que d'être sujets à l'institutionnalisation, ces mouvements sont porteurs d'innovations institutionnelles ${ }^{30}$. L'intérêt de ces analyses réside précisément dans la reconnaissance d'une remise en cause de l'ordre social. Alors que l'approche de la mobilisation des ressources nous force à concevoir l'action collective à l'intérieur d'une structure sociopolitique donnée dans le cadre étroit d'une logique d'intérêts, la perspective actionnaliste envisage les nouveaux mouvements sociaux comme un élément constitutif des transformations sociales actuelles. Ceci ne signifie pas que l'approche de la mobilisation des ressources n'a aucune pertinence ou que ses travaux sont sans valeur. À l'instar de

28 Alberto Melucci, « Société en changement et nouveaux mouvements sociaux », Sociologie et sociétés, vol. 10, $\mathrm{n}^{\circ}$ 2, 1978, p. 37-53 ; Alberto Melucci, "Mouvements sociaux, mouvements post-politiques », Revue Internationale d'Action Communautaire, $\mathrm{n}^{\circ}$ 10/50, 1983, p. 13-30; Alberto Melucci, «Qu'y a-t-il de nouveau dans les "Nouveaux mouvements sociaux" ? ", in A. Sales et L. Maheu (dir.), La recomposition du politique, PUM et L’Harmattan, Montréal et Paris, 1991, p. 129-162; Alberto Melucci, « Identité et changement : le défi planétaire de l'action collective ", in Juan-Luis Klein et al. (dir.), Au-delà du néolibéralisme, quel rôle pour les mouvements sociaux, PUQ, Sainte-Foy, 1997, p. 9-20.

29 Claus Offe, Les démocraties modernes à l'épreuve, L'Harmattan, Paris et Montréal, 1997 ; Claus Offe, « New social movements : challenging the boundaries of institutional politics ", Social Research, vol. 52, n 4, 1985, p. $817-868$.

30 Klaus Eder, The institutionalization of social movement. Towards a new theoretical problematic in socialmovement analysis ?, European University Institute, Florence, 1993. 
Neveu, nous sommes d'avis que la succession et la confrontation des théories sur les mouvements sociaux ont bel et bien produit un socle théorique solide "laissant "au creux de la main" un précieux capital de savoirs et d'instruments d'analyse $e^{31} »$. Seulement, envisagée dans un cadre tourainien, la perspective de l'école de mobilisation des ressources semble relever du niveau organisationnel. Or, nous arguons que la problématique environnementale constitue un enjeu historique, c'est-àdire un enjeu au cœur de la reconfiguration des rapports sociaux inhérente à l'avènement d'une société post-industrielle. Elle doit donc être appréhendée aux niveaux historique et institutionnel avant de faire l'objet d'une analyse organisationnelle car ces niveaux la précédent dans l'analyse ${ }^{32}$.

Plus englobant, le cadre actionnaliste s'arrime aisément à la théorie de la régulation comme l'illustrent les travaux de Bélanger et Lévesque ${ }^{33}$. En effet, l'articulation des sphères économique et sociale postulée par les régulationnistes trouve écho dans la distinction structurelle tourainienne de l'action sociale qui assujettit l'ordre organisationnel à l'ordre institutionnel, lequel est à son tour déterminé par les luttes entre les acteurs sociaux. Loin d'une perspective néoclassique, les deux cadres analytiques envisagent les institutions comme le résultat de compromis entre des acteurs dominants et des acteurs dominés ; ils insistent sur le fait que le conflit social n'est jamais entièrement résolu dans ces compromis, et nourrit des tensions qui peuvent mener à leur rupture ${ }^{34}$. Le concept de régulation sociale et celui de la crise envisagée comme période ouverte renvoient à la double dimension tourainienne de production et reproduction de la société ; une reproduction des rapports de domination qui s'accompagne d'une production de la société à travers le conflit des acteurs sociaux pour le contrôle de l'historicité. Le concept d'historicité pour sa part n'est pas sans lien avec celui de paradigme sociétal, ou de vision du progrès propre au cadre régulationniste. On peut aussi voir des correspondances entre le régime d'accumulation régulationniste et le concept d'investissement avancé par Touraine. L'intérêt des thèses de Touraine pour nos recherches réside également

31 Érik Neveu, op. cit., p. 38.

32 Alain Touraine, 1978, op. cit.

33 Paul R. Bélanger et Benoît Lévesque, op. cit.

34 Alain Lipietz, « Bases pour une alternative démocratique », Colloque GRETSE, Les formes modernes de la démocratie, $\mathrm{n}^{\circ}$ 8910, Montréal, 1-3 mars 1989 ; Alain Touraine, 1973, op. cit. 
dans le fait que le mouvement central qu'il anticipe s'articule vraisemblablement autour des groupes écologistes. Même en écartant l'idée d'une structure sociale bipolaire, qui semble moins appropriée dans le contexte actuel, en faveur d'une conception tri ou multipolaire des mouvements sociaux, le cadre analytique tourainien demeure pertinent pour intégrer une dimension sociologique plus réaliste au cadre régulationniste.

Confrontée à ce cadre socio-économique, les approches économiques de la problématique environnementale qui traduisent une perspective essentiellement technique de la modernisation écologique paraissent également relever du niveau organisationnel. Si bien que leurs analyses n'épuisent guère la dimension sociale et institutionnelle de la modernisation qui nécessite une compréhension des dynamiques proprement sociales à l'œuvre dans le domaine de l'environnement. Or, c'est à ces niveaux que se cristalliseront les transformations structurelles qui, comme de nouvelles règles du jeu, viendront affecter les pratiques organisationnelles. Mais il importe également de saisir que derrière les enjeux strictement environnementaux, des classes ou des acteurs sociaux sont en lutte pour le contrôle de l'historicité, certes, mais aussi pour gagner une meilleure place dans les processus décisionnels aux niveaux politiques et organisationnels. La problématique environnementale est une occasion de revendiquer ce pouvoir décisionnel dans le cadre d'une nouvelle gouvernance plus démocratique. De façon parallèle, la matérialité de la crise écologique se traduira par des charges susceptibles de provoquer de nouvelles tensions ou d'approfondir les conflits sociaux déjà présents. Dans la mesure où l'on admet l'idée du compromis, c'està-dire qu'un acteur dominant imposera son dispositif tout en faisant des concessions, il nous est apparu particulièrement intéressant de nous pencher sur la classe dirigeante, soit l'élite économique, plutôt que sur le mouvement écologiste qui a déjà fait l'objet de maintes études. De cette façon, nous répondons un peu à l'ambition qu'avait Touraine de compléter son analyse des nouveaux mouvements sociaux par celle de la classe dirigeante ${ }^{35}$. Mais surtout, en nous concentrant sur cette classe et ses représentations de la problématique environnementale et de l'organisation politico-économique de la société ainsi que sa conception 
du progrès social, nous croyons être mieux en mesure d'anticiper la forme et l'orientation d'un compromis potentiel et de comprendre la dynamique de transformation des institutions économiques induite par la problématique environnementale.

\section{Les configurations possibles de la modernisation écologique des institutions économiques}

En nous inspirant du modèle de Sklair et sans réduire la structure sociale à une configuration bipolaire, on peut avancer que la problématique environnementale est le terrain d'une confrontation entre une classe économique et une «classe » écologiste ${ }^{36}$. Dans la mesure où l'on admet une certaine sensibilisation des gens d'affaires à la question environnementale, l'écologisme consumériste que Sklair présente comme une récupération peut aussi être envisagé comme un compromis. Sur la base de ce modèle, et en ayant à l'esprit les réflexions qui précèdent, on peut donc avancer l'idée d'un compromis post-fordiste consumériste dématérialisé, probablement transitoire, dont la forme et la viabilité dépendront des répercussions matérielles de la crise écologique et de la capacité de transférer les externalités en périphérie. Le caractère global des atteintes à l'environnement dont les manifestations seront de plus en plus lourdes de même que la dépendance matérielle du Nord vis-à-vis du Sud constitueront des défis majeurs au maintien d'un tel compromis. À l'intérieur de cette perspective globale, on peut constater l'entrée de l'environnement dans le champ de l'historicité dont témoignent la reconnaissance de l'élite économique et le déplacement du paradigme sociétal industriel vers un nouvel idéal que pourrait traduire le concept de développement durable. Selon nos recherches, le paradigme sociétal qui semble émerger du discours des dirigeants comporte d'importantes ruptures avec le paradigme précédent ${ }^{37}$. Le progrès ne s'incarne plus dans l'industrialisation mais bien dans une économie hautement technologique peu intensive sur le plan écologique. Bien qu'encore indéfinis, les modes de régulation sont envisagés au niveau

36 Leslie Sklair, « Global sociology and global environmental change », in Michael Redclif et Ted Benton (dir.), Social theory and the global environment, Routledge, Londres et New York, 1994, p. 205-227.

37 En 1999 et 2000, nous avons interrogé une vingtaine de hauts dirigeants économiques issus du palmarès Top 50 des gens les plus influents au Québec dans le cadre d'entretiens semi-dirigés d'une durée d'une à deux heures. Voir Éthique et développement économique : le discours des dirigeants d'entreprises sur l'environnement, Département de sociologie, Université du Québec à Montréal, décembre 2000. 
international à travers une compétition mondialisée ou une réglementation de type politique assurée par des institutions mondiales encore virtuelles. Tout en reconnaissant les limites écologiques de la production économique, ce paradigme repose sur une conception consumériste du bien être où la croissance économique témoigne toujours d'un accroissement de la richesse. En conséquence, on peut penser que les compromis susceptibles de se nouer entre l'élite économique et les écologistes aura tendance à se rapprocher du modèle écologiste consumériste avancé par Leslie Sklair. Compte tenu du paradigme en émergence et des compromis sociaux auquel il est susceptible de donner lieu, on peut entrevoir quel accueil sera réservé aux deux propositions des principaux courants en économie de l'environnement.

En ce qui concerne l'argumentation des économistes écologistes tout d'abord, la croissance zéro semble difficilement recevable dans la mesure où elle est en flagrante contradiction avec un paradigme sociétal verrouillé dans l'idéal d'une croissance économique mondialisée. On doit néanmoins souligner les tentatives de renouvellement de la comptabilité nationale qui questionnent la signification des indices de croissance économique au chapitre du niveau de développement réel d'un pays ou d'une population. La question de l'internalisation des coûts est plus délicate. Signalons tout d'abord que les dirigeants sont imperméables à l'argumentaire de l'internalisation des coûts écologiques et pour la plupart d'entre eux, la protection de l'environnement apparaît non pas comme une externalité mais comme un coût supplémentaire imposé par un État qui n'a pas la légitimité d'entraver la production de richesse en minant la capacité compétitive de ses entreprises. L'internalisation des coûts à l'échelle nationale est donc grandement limitée par le contexte de la mondialisation car elle correspond à une anti-stratégie pour des entreprises forcées de rentrer en compétition sur les marchés internationaux. En fait, on peut s'attendre à ce que les stratégies d'internalisation des coûts environnementaux soient largement tributaires des rapports de pouvoirs existant sur la scène internationale. Comme le montrent Muradian et Martinez-Alier, on assiste déjà à un transfert des coûts environnementaux vers le Sud et à une intensification écologique de ses économies ${ }^{38}$. Ceci permet aux économies dominantes de procéder à une dématérialisation progressive de leurs activités et d'orienter leur production vers des secteurs moins intensifs sur le plan écologique. Si bien que l'internalisation des coûts pourrait accompagner une conversion des économies occidentales, mais ne la 
précédera pas contrairement à ce que laissent entendre les économistes de l'environnement.

Par ailleurs, on peut avancer que l'internalisation des coûts ne sera pas nécessairement portée par les pouvoirs publics. Les pressions exercées par les groupes à l'égard des installations polluantes se traduisent, aux yeux des dirigeants eux-mêmes, par des coûts assimilables à n'importe quel autre coût de production. On peut également penser qu'à moyen terme, les principaux producteurs d'externalités environnementales seront de plus en plus marginalisés dans la mesure où les dirigeants eux-mêmes acceptent de moins en moins que le développement et la croissance économique se fassent au prix d'une dégradation de l'environnement. En ce qui concerne la consommation, nos recherches semblent confirmer les conclusions de Sklair selon lequel on devrait s'acheminer vers un compromis consumériste. La modernisation écologique ne s'appuiera donc pas sur une transformation radicale du schéma de sur-consommation mais peut-être sur une dématérialisation de la consommation, bien que cette tendance ne soit pas encore observable pour le moment. On peut donc avancer que le compromis post-fordiste écologique pourrait reposer sur un triple processus de dématérialisation : une transformation de la consommation, un transfert des coûts écologiques vers le Sud et une dématérialisation technologique réservée à quelques secteurs de pointe triés sur le volet et réalisée avec le concours de l'État.

Nous avons cherché dans cet article à répondre aux propositions de modernisation écologique avancées par les économistes de l'environnement en révélant les déterminants sociaux de cette modernisation. Alors que les modèles économiques font abstraction du caractère construit de la crise écologique, mais également du système économique, nous avançons à l'instar de Touraine et des régulationnistes, que les formes institutionnelles résultent de compromis sociaux formés sur la base d'un paradigme sociétal hégémonique. On ne peut donc penser la modernisation écologique des institutions économiques sans prendre en considération les rapports sociaux et leur dynamique, et sans comprendre les représentations des acteurs dominants qui tenteront d'imposer un dispositif conforme à leurs intérêts, tout en le faisant reconnaître comme légitime par les autres acteurs sociaux.

38 Ronald Muradian et Joan Martinez-Alier, «Trade and the environment : from a "southern" perspective ", communication à Transitions towards a sustainable Europe. Ecology-economy-policy, 3rd Biennal Conference of the European Society for Ecological Economics, Vienne, 3-6 mai 2000. 